\title{
CENTRAL EXTENSIONS OF CURRENT ALGEBRAS
}

\author{
PAUL ZUSMANOVICH
}

\begin{abstract}
The second cohomology group of Lie algebras of kind $L \otimes U$ with trivial coefficients is investigated, where $L$ admits a decomposition with onedimensional root spaces and $U$ is an arbitrary associative commutative algebra with unit. This paper gives a unification of some recent results of $C$. Kassel and A. Haddi and provides a determination of central extensions of certain modular semisimple Lie algebras.
\end{abstract}

\section{INTRODUCTION}

The main result of this paper (Theorem 1.3) gives the expression of the second cohomology group of a Lie algebra $L \otimes U$ with trivial coefficients (denoted by $H^{2}(L \otimes U)$ ), where $L$ belongs to the class of Lie algebras referred to as Block algebras (for the definition, see below), and $U$ is a commutative associative algebra with unit, through the first-order cyclic cohomology $H C^{1}(U)$ and the dual $U^{*}$. Since the classical Lie algebras, Zassenhaus algebras $W_{1}(n)$, and infinite-dimensional Witt algebras $W$ are Block algebras, we obtain as corollaries the values of $H^{2}(L \otimes U)$ in all these cases. In the second section of this paper the cohomology groups $H^{2}\left(W_{1}(n) \otimes U\right)$ are computed by a different method, based on the presentation of $W_{1}(n)$ as a deformation of tensor product of $W_{1}(1)$ and divided power algebra. Notice that $W_{1}(\infty)$ is not a Block algebra and hence this case is not covered by the previous theorem. The third section contains an application of the preceding results to the computation of central extensions of certain modular semisimple Lie algebras.

Note that earlier C. Kassel [9] and A. Haddi [8] computed the second homology group $H_{2}(L \otimes U)$, where $L$ is classical or $W$ and its subalgebra $W_{1}$ respectively. Their method is quite different from ours and is based on the idea of universal central extensions. Our main theorem may be considered as a generalization and unification of Kassel's and Haddi's results.

All algebras are defined over a field $\mathbb{F}$ of characteristic $p \neq 2,3$, if the otherwise condition is not stated. Remember that $U$ denotes an arbitrary associative commutative algebra with a unit 1 .

\section{THE MAIN THEOREM AND COROLLARIES}

Let $T$ be a torus in a Lie algebra $L$. Assume that there exists a root space decomposition of $L$ with respect to the $T$-action:

Received by the editors April 18, 1990 and, in revised form, September 4, 1990.

1980 Mathematics Subject Classification (1985 Revision). Primary 17B56; Secondary 17B50, 17B20, $13 \mathrm{D} 03$. 


$$
L=T \oplus \bigoplus_{\alpha \in R} L_{\alpha} .
$$

Assume, moreover, that all root spaces are one-dimensional: $L_{\alpha}=\mathbb{F} e_{\alpha}$ and $\alpha\left(\left[L_{\alpha}, L_{-\alpha}\right]\right) \neq 0$ for all $\alpha \in R$. Set $h_{\alpha}=\left[e_{-\alpha}, e_{\alpha}\right],\left[e_{\alpha}, e_{\beta}\right]=N_{\alpha, \beta} e_{\alpha+\beta}$, $N_{\alpha, \beta} \in \mathbb{F}$. The next assumption concerning the structure of $L$ is $[L, L]=L$. This is equivalent to $T=\sum \mathbb{F} h_{\alpha}$. Let $\left\{h_{\alpha} \mid \alpha \in B\right\}, B \subseteq R$, be a basis in $T$.

Lie algebras $L$ satisfying all these properties will be called Block algebras. In [1] R. E. Block showed that all finite-dimensional Block algebras over a perfect field of characteristic $p>5$ with $Z(L)=0$ are direct sums of simple classical Lie algebras and Albert-Zassenhaus algebras.

In the sequel, $L$ will denote a Block algebra except in Lemma 1.1 and Lemma 3.1 , where $L$ is arbitrary.

Our cohomological notions and notations are standard (cf. [7] for Lie algebra cohomology and [5] for cyclic cohomology). When we consider the Lie algebra cohomology, we use the standard cochain complex. Notice that since $U$ is commutative, the first-order cyclic cohomology $H C^{1}(U)$ is exactly the module of all skew-symmetric functions $F \in(U \otimes U)^{*}$ such that

$$
F(u v, w)+F(w u, v)+F(v w, u)=0 .
$$

If $\mathscr{D}$ is a subalgebra in $\operatorname{Der}(U)$, the module of $\mathscr{D}$-invariant cyclic cohomology $H C^{1}(U)^{\mathscr{D}}$ consists of cocycles satisfying

$$
F(D(u), v)+F(u, D(v))=0
$$

for all $d \in \mathscr{D}$.

Recall that the Lie algebra structure on $L \otimes U$ is defined by $[x \otimes u, y \otimes v]=$ $[x, y] \otimes u v$.

Now compute $H^{2}(L \otimes U)$.

The following lemma is probably one of the most useful results for computation of Lie algebra cohomology groups.

Lemma 1.1. (Cf. [7, Theorem 1.5.2]). Let $L$ be a Lie algebra, and let $T$ be a torus in $L$ such that there exists a root space decomposition (1). Then for each cohomology class $[\Phi] \in H^{k}(L)$ there exists a representative cocycle $\Phi$ such that $\Phi\left(L_{\alpha_{1}}, \ldots, L_{\alpha_{k}}\right)=0$ if $\alpha_{1}+\cdots+\alpha_{k} \neq 0$.

Lemma $1.2 H^{2}(\operatorname{sl}(2) \otimes U) \cong H C^{1}(U)$.

If we choose a basis $\{e, f, h\}$ in $\mathrm{sl}(2)$ with multiplication table

$$
[h, e]=-\alpha e, \quad[h, f]=\alpha f, \quad[e, f]=h
$$

for certain $\alpha \in \mathbb{F} \backslash\{0\}$, then the basic cocycles may be chosen as follows:

$$
\begin{gathered}
h \otimes u \wedge h \otimes v \mapsto-\alpha F(u, v), \\
e \otimes u \wedge f \otimes v \mapsto F(u, v), \\
\text { other combinations } \mapsto 0
\end{gathered}
$$

where $F \in H C^{1}(U)$.

Proof. Straightforward computations or reference to Kassel [9]. Perhaps it should only be remarked that the coboundary cyclic (= Hochschild) cohomology condition arises from the Jacobi identity. 
Theorem 1.3. $H^{2}(L \otimes U) \cong\left(\bigoplus_{\alpha \in R} H C^{1}(U)_{\alpha}\right) / I \oplus\left(\bigoplus_{\alpha \in R} U_{\alpha}^{*}\right) / J$ where $H C^{1}(U)_{\alpha}$ $\cong H C^{1}(U), U_{\alpha}^{*} \cong U^{*}$ for any $\alpha \in R, I$ is the linear span of the following elements:

$$
\alpha(h) F_{\alpha}-\sum_{\beta \in B} \lambda_{\alpha \beta} \beta(h) F_{\beta}, \quad \alpha\left(h_{\beta}\right) F_{\alpha}-\beta\left(h_{\alpha}\right) F_{\beta}, \quad N_{\beta,-\alpha-\beta} F_{\alpha}+N_{\alpha,-\alpha-\beta} F_{\beta}
$$

for all $h_{\alpha}, h \in T$, where $h_{\alpha}=\sum_{\beta \in B} \lambda_{\alpha \beta} h_{\beta}$ is the linear expression of $h_{\alpha}$ in terms of the basis $\left\{h_{\beta} \mid \beta \in B\right\}$, and for all $\alpha, \beta \in R$, where $F_{\alpha}$ is an arbitrary element in $H C^{1}(U)_{\alpha} . J$ is the linear span of the elements

$$
\begin{array}{r}
N_{\beta, \gamma} G_{\alpha}+N_{\gamma, \alpha} G_{\beta}+N_{\alpha, \beta} G_{\gamma}, \quad \alpha, \beta, \gamma \in R, \alpha+\beta+\gamma=0 ; \\
G_{-\alpha}+G_{\alpha}, \alpha \in R ; G_{\alpha}, \alpha \in B,
\end{array}
$$

for all $G_{\alpha} \in U_{\alpha}^{*}$.

Basic cocycles may be chosen as follows:

$$
\begin{gathered}
h_{\alpha} \otimes u \wedge h_{\beta} \otimes v \mapsto-\alpha\left(h_{\beta}\right) F_{\alpha}(u, v), \quad \alpha, \beta \in B, \\
e_{-\alpha} \otimes u \wedge e_{\alpha} \otimes v \mapsto F_{\alpha}(u, v)+G_{\alpha}(u v), \quad \alpha \in R, \\
\text { other combinations } \mapsto 0 .
\end{gathered}
$$

Proof. Obviously for any root $\alpha$ it is possible to find a complement $Z_{\alpha}$ of $\mathbb{F} h_{\alpha}$ in $T$ such that $\left[Z_{\alpha}, e_{\alpha}\right]=\left[Z_{\alpha}, e_{-\alpha}\right]=0 . T \oplus \mathbb{F} e_{-\alpha} \oplus \mathbb{F} e_{\alpha}$ is a subalgebra in $L$ which is isomorphic to an (unessential) central extension of $\operatorname{sl}(2)$. Since

$H^{2}\left(\left(T \oplus \mathbb{F} e_{-\alpha} \oplus \mathbb{F} e_{\alpha}\right) \otimes U\right) \cong H^{2}\left(Z_{\alpha} \otimes U\right)+H^{2}\left(\left(\mathbb{F} h_{\alpha} \oplus \mathbb{F} e_{-\alpha} \oplus \mathbb{F} e_{\alpha}\right) \otimes U\right)$,

by Lemma 1.2 we get

$$
H^{2}\left(\left(T \oplus \mathbb{F} e_{-\alpha} \oplus F e_{\alpha}\right) \otimes U\right) \cong C^{2}\left(Z_{\alpha} \otimes U\right)+H C^{1}(U) .
$$

Hence for arbitrary $\Phi \in Z^{2}(L \otimes U)$ and $\alpha \in R$ we have

$$
\begin{gathered}
\Phi\left(h_{\alpha} \otimes u, h_{\alpha} \otimes v\right)=-\alpha\left(h_{\alpha}\right) F_{\alpha}(u, v), \\
\Phi\left(e_{-\alpha} \otimes u, e_{\alpha} \otimes v\right)=F_{\alpha}(u, v)+G_{\alpha}(u v), \\
\Phi\left(h_{\alpha} \otimes U, Z_{\alpha} \otimes U\right)=0,
\end{gathered}
$$

for certain $F_{\alpha} \in H C^{1}(U)$ and $G_{\alpha} \in U^{*}$.

For any $h \in T, h-\left(\alpha(h) / \alpha\left(h_{\alpha}\right)\right) h_{\alpha} \in Z_{\alpha}$. Hence

$$
\Phi\left(h_{\alpha} \otimes u,\left(h-\left(\alpha(h) / \alpha\left(h_{\alpha}\right)\right) h_{\alpha}\right) \otimes v\right)=0
$$

and

$$
\Phi\left(h_{\alpha} \otimes u, h \otimes v\right)=\left(\alpha(h) / \alpha\left(h_{\alpha}\right)\right) \Phi\left(h_{\alpha} \otimes u, h_{\alpha} \otimes v\right)=-\alpha(h) F_{\alpha}(u, v) .
$$

Now suppose that in the last equality $h_{\alpha}=\sum_{\beta \in B} \lambda_{\alpha \beta} h_{\beta}$. Then

$$
\begin{aligned}
-\alpha(h) F_{\alpha}(u, v) & =\sum_{\beta \in B} \lambda_{\alpha \beta} \Phi\left(h_{\beta} \otimes u, h \otimes v\right) \\
& =-\sum_{\beta \in B} \lambda_{\alpha \beta} \beta(h) F_{\beta}(u, v) .
\end{aligned}
$$

This implies the defining relations for $I$ of the first kind. Further, substituting in (5) $h=h_{\beta}$, we obtain (2) and (because of the skew-symmetry of $\Phi$ ) the defining relations for $I$ of the second kind:

$$
\alpha\left(h_{\beta}\right) F_{\alpha}(u, v)=\beta\left(h_{\alpha}\right) F_{\beta}(u, v) .
$$


The cocycle equation $d \Phi=0$ for the triple $e_{\alpha} \otimes u, e_{\beta} \otimes v, e_{\gamma} \otimes w, \alpha+\beta+\gamma=0$, is equivalent to the relation $N_{\alpha, \beta} F_{\gamma}(u v, w)+N_{\alpha, \beta} G_{\gamma}(u v w)+($ simultaneous cyclic permutations of $(\alpha, \beta, \gamma)$ and $(u, v, w))=0$. Substituting here $v=$ $w=1$, we obtain $N_{\beta, \gamma} G_{\alpha}(u)+N_{\gamma, \alpha} G_{\beta}(u)+N_{\alpha, \beta} G_{\gamma}(u)=0$ (which is equivalent to the defining relations for $J$ of the first kind) and hence

$$
N_{\beta, \gamma} F_{\alpha}(v w, u)+N_{\gamma, \alpha} F_{\beta}(w u, v)+N_{\alpha, \beta} F_{\gamma}(u v, w)=0 .
$$

Substituting in the last equality $w=1$ we get the defining relations for $I$ of the third kind:

$$
N_{\beta,-\alpha-\beta} F_{\alpha}(v, u)+N_{-\alpha-\beta, \alpha} F_{\beta}(u, v)=0 .
$$

Since $T \otimes 1$ is a torus in $L \otimes U$, by Lemma 1.1 we may assume

$$
\begin{gathered}
\Phi\left(e_{\alpha} \otimes U, e_{\beta} \otimes U\right)=0 \quad \text { if } \alpha+\beta \neq 0, \\
\Phi\left(T \otimes U, e_{\alpha} \otimes U\right)=0,
\end{gathered}
$$

which implies (4).

Now put $\omega \in C^{1}(L \otimes U)$ as follows:

$$
\omega\left(h_{\alpha} \otimes u\right)=G_{\alpha}(u), \quad \alpha \in B ; \quad \omega\left(e_{\alpha} \otimes U\right)=0, \quad \alpha \in R .
$$

Replacing $\Phi$ by $\Phi-d \omega$, we obtain a cocycle which satisfies all previous equalities and, moreover, $G_{\alpha}=0, \alpha \in B$. Finally, the relation $G_{\alpha}+G_{-\alpha}=0$ obviously follows from the skew-symmetry of $\Phi$.

It remains to prove only that $\Phi$ described in the theorem is really a cocycle and that all such cocycles are independent. This is achieved by an easy argument which mainly repeats the previous one and is left to the reader.

We keep the symbol $\mathfrak{g}$ for classical Lie algebras, that is, for Lie algebras obtained from integral forms of complex classical simple Lie algebras by tensoring on $\mathbb{F}$. Let $R$ be a root system of $\mathfrak{g}, B$ be a basis, $\left\{h_{\alpha}, \alpha \in B ; e_{\alpha}, \alpha \in R\right\}$ be a Chevalley basis of $\mathfrak{g}$, and let $\langle\cdot, \cdot\rangle$ denote a scalar product in $R$.

Corollary 1.4 [9]. $H^{2}(\mathfrak{g} \otimes U) \cong H C^{1}(U)$.

Basic cocycles may be chosen as follows:

$$
\begin{gathered}
h_{\alpha} \otimes u \wedge h_{\beta} \otimes v \mapsto-\frac{2\langle\alpha, \beta\rangle}{\langle\alpha, \alpha\rangle\langle\beta, \beta\rangle} F(u, v), \quad \alpha, \beta \in B, \\
e_{-\alpha} \otimes u \wedge e_{\alpha} \otimes v \mapsto \frac{1}{\langle\alpha, \alpha\rangle} F(u, v), \quad \alpha \in R, \\
\text { other combinations } \mapsto 0,
\end{gathered}
$$

where $F \in H C^{1}(U)$.

Proof. There is the following property of the structure constants:

$$
\frac{N_{\alpha, \beta}}{\langle\gamma, \gamma\rangle}=\frac{N_{\beta, \gamma}}{\langle\alpha, \alpha\rangle}=\frac{N_{\gamma, \alpha}}{\langle\beta, \beta\rangle} \quad \text { if } \alpha+\beta+\gamma=0
$$

(cf. [4, Chapter VIII, §2, Exercise 4]). This property and the defining relations for $J$ of the first kind implies

$$
G_{-\alpha-\beta}=-\frac{\langle\alpha, \alpha\rangle}{\langle\alpha+\beta, \alpha+\beta\rangle} G_{\alpha}-\frac{\langle\beta, \beta\rangle}{\langle\alpha+\beta, \alpha+\beta\rangle} G_{\beta}, \quad \alpha, \beta, \alpha+\beta \in R .
$$


But $G_{\alpha}=0$ for all $\alpha \in B$ and, consequently, $G_{\alpha}=0$ for all negative roots $\alpha$. The defining relations for $J$ of the second kind yield $G_{-\alpha}=-G_{\alpha}$ and hence $G_{\alpha}=0$ for all positive roots $\alpha$.

Further, (7) together with the defining relations for $I$ of the third kind gives $\langle\alpha, \alpha\rangle F_{\alpha}=\langle\beta, \beta\rangle F_{\beta}, \alpha, \beta \in R$. Hence

$$
F_{\alpha} \frac{1}{\langle\alpha, \alpha\rangle} F, \quad \alpha \in R,
$$

for certain $F \in H C^{1}(U)$. Since for $\alpha, \beta \in R, \alpha\left(h_{\beta}\right)=2\langle\alpha, \beta\rangle /\langle\beta, \beta\rangle$, the right-hand side of formula (2) takes the form

$$
-\frac{2\langle\alpha, \beta\rangle}{\langle\alpha, \alpha\rangle\langle\beta, \beta\rangle} F(u, v)
$$

which agrees with (6). Finally, it is easy to check that under condition (8) the defining relations for $I$ of the first and second kinds always holds.

Now recall the definition of the modular Zassenhaus algebra $W_{1}(n)$. It may be defined in two ways. Firstly as an algebra with basis $\left\{f_{\alpha} \mid \alpha \in \mathbb{G F}\left(p^{n}\right)\right\}$ and Lie bracket given by

$$
\left[f_{\alpha}, f_{\beta}\right]=(\beta-\alpha) f_{\alpha+\beta} .
$$

Secondly, as an algebra with basis $\left\{e_{i} \mid-1 \leq i \leq p^{n}-2\right\}$ and bracket

$$
\left[e_{i}, e_{j}\right]=\left(\left(\begin{array}{c}
i+j+1 \\
j
\end{array}\right)-\left(\begin{array}{c}
i+j+1 \\
i
\end{array}\right)\right) e_{i+j} \text {. }
$$

There are some related infinite-dimensional algebras. If we assume that the indices in formula (9) run over all integer, then we obtain the infinite-dimensional Witt algebra $W$ (considered over a field of characteristic zero). If we assume that the indices in formula (10) run from -1 to infinity, we obtain the infinitedimensional algebra $W_{1}(\infty)$ (considered over a field of positive characteristic). Obviously $W_{1}(n), n<\infty$, and $W$ are Block algebras.

Corollary 1.5. (i) $(p>3, n<\infty) H^{2}\left(W_{1}(n) \otimes U\right) \cong H^{2}\left(W_{1}(n)\right) \otimes U^{*}$.

(ii) [8] $(p=0) \quad H^{2}(W \otimes U) \cong H^{2}(W) \otimes U^{*}$.

Proof. (i). Choose a basis $\left\{f_{\alpha} \mid \alpha \in \mathbb{G F}\left(p^{n}\right)\right\}$. We have (in terms of Block algebras): $h_{\alpha}=2 f_{0}$ for all $\alpha \in R=\mathbb{G F}\left(p^{n}\right) \backslash\{0\}, \alpha\left(f_{0}\right)=\alpha, N_{\alpha, \beta}=\beta-\alpha$. Apply Theorem 1.3. The defining relations for $I$ of the second and third kinds give $\alpha F_{\alpha}=\beta F_{\beta}$ and $(\alpha+2 \beta) F_{\alpha}+(2 \alpha+\beta) F_{\beta}=0$. These equalities evidently imply $F_{\alpha}=0$ for all $\alpha$. The defining relations for $J$ give

$$
(\beta-\alpha) G_{-\alpha-\beta}-(\alpha+2 \beta) G_{\alpha}+(2 \alpha+\beta) G_{\beta}=0, \quad G_{-\alpha}=-G_{\alpha}, \quad G_{1}=0 .
$$

Then we proceed, as in Block's paper [2, p. 1449], to obtain $G_{\alpha}=\left(\alpha-\alpha^{3}\right) G$ for certain $G \in U^{*}$. Together with a result of Block [2, Theorem 5.1] this completes the proof.

(ii) Quite analogously.

Corollaries 1.4 and 1.5 show that the "cyclic cohomology" summand in our main formula (Theorem 1.3) arises from the "classical" properties of Block algebras, while the "dual" summand arises from "Zassenhaus" = ("nonclassical") ones. Of course, Theorem 1.1 may be applied to compute $H^{2}(L \otimes U)$ for other 
classes of Block algebras, e.g. Albert-Zassenhaus or Virasoro (both modular and nonmodular).

\section{Central extensions of $W_{1}(n) \otimes U$}

Define a Lie algebra $\mathfrak{L}(U, D)$, where $D \in \operatorname{Der}(U)$, as the vector space $W_{1}(1) \otimes U$ with Lie bracket given by $\{x, y\}=[x, y]+\Psi(x, y)$ where $[\cdot, \cdot]$ is the ordinary Lie bracket in $W_{1}(1) \otimes U$ and

$$
\Psi\left(e_{i} \otimes u, e_{j} \otimes v\right)=\left\{\begin{array}{l}
e_{p-2} \otimes v D(u)-u D(v), \quad i=j=-1, \\
0, \quad \text { otherwise. }
\end{array}\right.
$$

Now the notion of divided power algebra $\mathscr{O}_{1}(n)$ is necessary. It is defined as an associative commutative algebra over a field of characteristic $p>0$ with basis $\left\{x^{i} \mid 0 \leq i<p^{n}\right\}$ and multiplication $x^{i} x^{j}=\left(\begin{array}{c}i+j \\ j\end{array}\right) x^{i+j}$ (the case $n=$ $\infty$ is also included). It is known that $\mathscr{O}_{1}(n)$ is isomorphic to the truncated polynomial algebra $\mathscr{O}_{n}=\mathbb{F}\left[x_{1}, \ldots, x_{n}\right] /\left(x_{1}^{p}, \ldots, x_{n}^{p}\right)$. We reserve the special notation $\partial$ for the derivation of $\mathscr{O}_{1}(n)$ defined by $\partial\left(x^{i}\right)=x^{i-1}$.

The following remarkable observation is due to M. I. Kuznetsov [10]: $W_{1}(n)$ is isomorphic to a certain deformation of $W_{1}(1) \otimes \mathscr{O}_{1}(n-1)$. Namely, $W_{1}(n)$ is isomorphic to the Lie algebra $\mathfrak{L}\left(\mathscr{O}_{1}(n-1),-\partial\right)$. This isomorphism is given by $e_{i} \otimes x^{k} \mapsto e_{p k+i}$ (the $e_{i}$ 's on the left-hand side belong to $W_{1}(1)$, while the ones on the right-hand side belong to $\left.W_{1}(n)\right)$.

Theorem 2.1. $H^{2}(\mathfrak{L}(U, D)) \cong\left\{G \in U^{*} \mid D \circ G=0\right\}$.

The basic cocycles may be chosen as follows:

$$
e_{i} \otimes u \wedge e_{j} \otimes v \mapsto \delta_{i+j, 0}(-1)^{i} G(v D(u))+\delta_{i+j, p}(-1)^{i} G(u v)
$$

Proof. It is a routine task to verify that cochains determined by formula (11) are cocycles, and we leave it again to the reader.

Let $\Phi \in Z^{2}(\mathfrak{L}(U, D))$. Since $e_{0} \otimes \mathbb{F}$ is a torus in $\mathfrak{L}(U, D)$ and the corresponding root spaces are $\mathfrak{L}_{i}=e_{i} \otimes U$, by Lemma 1.1 we may assume that

$$
\phi\left(e_{i} \otimes U, e_{j} \otimes U\right)=0 \quad \text { if } i+\dot{j} \neq 0 \text { or } p .
$$

The cocycle equation is equivalent to the two following equalities:

$$
\begin{aligned}
& N_{i, j} \Phi\left(e_{i+j} \otimes u v, e_{k} \otimes w\right) \\
& \quad+(\text { simultaneous cyclic permutations of }(i, j, k) \\
& \quad \text { and }(u, v, w))=0, \quad\{i, j, k\} \neq\{-1,-1,2\},
\end{aligned}
$$

and

$$
\begin{aligned}
\Phi\left(e_{-1} \otimes v, e_{1} \otimes u w\right)-\Phi\left(e_{-1} \otimes u, e_{1} \otimes v w\right) \\
-\Phi\left(e_{2} \otimes w, e_{p-2} \otimes v D(u)-u D(v)\right)=0 .
\end{aligned}
$$

In (13), $k=-1, i+j=p+1$ :

$$
\Phi\left(e_{i} \otimes u, e_{j-1} \otimes v w\right)+\Phi\left(e_{i-1} \otimes u w, e_{j} \otimes v\right)=0 .
$$

Substitute here $w=1$ :

$$
\Phi\left(e_{i} \otimes u, e_{j-1} \otimes v\right)=-\Phi\left(e_{i-1} \otimes u, e_{j} \otimes v\right), \quad i+j=p+1 .
$$

Substituting the last equality in the previous one, we obtain

$$
\Phi\left(e_{i-1} \otimes u, e_{j} \otimes v w\right)=\Phi\left(e_{i-1} \otimes u w, e_{j} \otimes v\right) .
$$


Substitute here $v=1$ :

$$
\Phi\left(e_{i-1} \otimes u, e_{j} \otimes w\right)=\Phi\left(e_{i-1} \otimes u w, e_{j} \otimes 1\right), \quad i+j=p+1 .
$$

(15) and (16) imply

$$
\Phi\left(e_{i} \otimes u, e_{j} \otimes v\right)=(-1)^{i} G(u v), \quad i+j=p,
$$

for certain $G \in U^{*}$.

In (14), substitute $v=1$ :

$$
\Phi\left(e_{-1} \otimes u, e_{1} \otimes w\right)=\Phi\left(e_{-1} \otimes 1, e_{1} \otimes u w\right)-G(w D(u)) .
$$

In (13), set $i=-1, j=0, k=1, w=1$ :

$$
\Phi\left(e_{0} \otimes u, e_{0} \otimes v\right)=\Phi\left(e_{-1} u v, e_{1} \otimes 1\right)-\Phi\left(e_{-1} \otimes u, e_{1} \otimes v\right) .
$$

Substituting the previous equality in the last one, we obtain $\Phi\left(e_{0} \otimes u, e_{0} \otimes v\right)=$ $-G(u D(v))$. Then the skew-symmetry of $\Phi$ implies $-G(u D(v))-G(v D(u))=$ 0 which is equivalent to $D \circ G=0$.

Now put $\omega \in C^{1}(\mathfrak{L}(U, D))$ as follows: $\omega\left(e_{0} \otimes u\right)=\Phi\left(e_{-1} \otimes 1, e_{1} \otimes u\right)$; $\omega\left(e_{i} \otimes U\right)=0, i \neq 0$. Then $d \omega\left(e_{-1} \otimes 1, e_{1} \otimes u\right)=\Phi\left(e_{-1} \otimes 1, e_{1} \otimes u\right)$. Hence if we take $\Phi-d \omega$ instead of $\Phi$, we obtain a cocycle satisfying all previous equalities and, moreover,

$$
\Phi\left(e_{-1} \otimes 1, e_{1} \otimes u\right)=0 .
$$

Collecting (12) and (17)-(20), we obtain formula (11).

It remains to prove that if $\Phi=d \omega$, where $\Phi$ is determined by formula (11) for an appropriate $G$, and $\omega \in C^{1}(\mathfrak{L}(U, D))$, then $G=0$. But this is obvious:

$$
G(u)=\Phi\left(e_{2} \otimes u, e_{p-2} \otimes 1\right)=d \omega\left(e_{2} \otimes u, e_{p-2} \otimes 1\right)=0 .
$$

Corollary 2.2. $(p>3) H^{2}\left(W_{1}(\infty) \otimes U\right)=0$.

Joint proof of Corollaries 1.5(i) and 2.2. According to the above-mentioned observation by Kuznetsov, $\mathfrak{L}\left(U \otimes \mathscr{O}_{1}(n-1),-1 \otimes \partial\right) \cong W_{1}(n) \otimes U$, and the isomorphism being given by $e_{i} \otimes u \otimes x^{k} \mapsto e_{p k+i} \otimes u$.

Let $n<\infty$. By Theorem 2.1

$$
\begin{aligned}
H^{2}\left(W_{1}(n) \otimes U\right) & \cong\left\{G \in\left(U \otimes \mathscr{O}_{1}(n)\right)^{*} \mid G\left(U \otimes\left\langle 1, x, x^{2}, \ldots, x^{p^{n-1}-2}\right\rangle\right)=0\right\} \\
& \cong U^{*} .
\end{aligned}
$$

Rewriting (11) in our case, we have

$$
\begin{aligned}
e_{p k+1} & \otimes v \wedge e_{p 1+j} \otimes u \mapsto-\left(\begin{array}{c}
k+l-1 \\
k-1
\end{array}\right) \delta_{i+j, 0}(-1)^{i} G\left(u v \otimes x^{k+l-1}\right) \\
& +\left(\begin{array}{c}
k+l \\
k
\end{array}\right) \delta_{i+j, p}(-1)^{i} G\left(u v \otimes x^{k+l}\right), \quad i, j=-1,0,1, \ldots, p-2
\end{aligned}
$$

Direct easy calculations show that it is exactly the formula

$$
e_{i} \otimes u \wedge e_{j} \otimes v \mapsto \delta_{i+j, p^{n}}(-1)^{i} G(u v) .
$$

Let $n=\infty$. Since $\partial\left(\mathscr{O}_{1}(\infty)\right)=\mathscr{O}_{1}(\infty)$, by Theorem $2 H^{2}\left(W_{1}(\infty) \otimes U\right)=$ 0 . 
Remark. The expression of basic cocycles of $H^{2}\left(W_{1}(n) \otimes U\right)$ in terms of the basis $\left\{e_{i}\right\}$ corresponds to the one for $H^{2}\left(W_{1}(n)\right)$ found by Dzhumadil'daev [6].

\section{Central extensions of MOdular Semisimple lie algebras}

According to [3], Lie algebras of type $\mathfrak{g} \otimes \mathscr{O}_{n}+1 \otimes \mathfrak{D}$ and $W_{1}(n) \otimes \mathscr{O}_{n}+1 \otimes \mathfrak{D}$, where $\mathfrak{D}$ is a subalgebra of $\operatorname{Der}\left(\mathscr{O}_{n}\right)$ such that $\mathscr{O}_{n}$ does not contain $\mathscr{D}$-invariant ideals, are one of the simplest but quite typical examples of modular semisimple Lie algebras. Preceding results allow us to determine their central extensions.

Lemma 3.1. Let $L+\mathfrak{D}$ be a semidirect product of Lie algebras, where $L$ is an ideal with $[L, L]=L$. Then $H^{2}(L+\mathfrak{D}) \cong H^{2}(L)^{\mathfrak{D}}+H^{2}(\mathfrak{D})$.

Proof. We may identify in an obvious manner $C^{2}(L+\mathfrak{D})$ with $C^{2}(L)+$ $(L \otimes \mathfrak{D})^{*}+C^{2}(\mathfrak{D})$. Let us see how this identification affects cocycles and coboundaries. Let $\Phi \in C^{2}(L+\mathfrak{D})$ and $\Phi=\Psi+\Upsilon+\Xi$ be the corresponding decomposition. The cocycle equation $d \Phi=0$ is equivalent to the following relations:

$$
\begin{gathered}
d \Psi(x \cdot y, z)=0, \\
\Upsilon([x, y], D)=\Psi([x, D], y)+\Psi(x,[y, D]), \\
\Upsilon\left(\left[x, D_{1}\right], D_{2}\right)-\Upsilon\left(\left[x, D_{2}\right], D_{1}\right)-\Upsilon\left(x,\left[D_{1}, D_{2}\right]\right)=0, \\
d \Xi\left(D_{1}, D_{2}, D_{3}\right)=0,
\end{gathered}
$$

for all $x, y, z \in L$ and $D, D_{1}, D_{2}, D_{3} \in \mathfrak{D}$. The second relation shows that $\Psi \in Z^{2}(L)^{\mathfrak{D}}:=\left\{\Psi \in Z^{2}(L) \mid \Psi([x, D], y)+\Psi(x,[y, D]) \in B^{2}(L) \quad \forall D \in\right.$ $\mathfrak{D}\}$ and, since $[L, L]=L, \Upsilon$ is fully determined by $\Psi$. Direct verification shows that the third relation follows from the two previous ones. So we have $Z^{2}(L+\mathfrak{D}) \cong Z^{2}(L)^{\mathfrak{D}}+Z^{2}(\mathfrak{D})$. Obviously $B^{2}(L+\mathfrak{D}) \cong B^{2}(L)+B^{2}(\mathfrak{D})$. The last two isomorphisms imply the assertion of the lemma.

Corollaries $1.4,1.5$, and the preceding lemma immediately imply the following proposition.

Proposition 3.2. (i) $H^{2}(\mathfrak{g} \otimes U+1 \otimes \mathfrak{D}) \cong H C^{1}(U)^{\mathfrak{D}}+H^{2}(\mathfrak{D})$.

(ii) $(p>3, n<\infty), H^{2}\left(W_{1}(n) \otimes U+1 \otimes \mathfrak{D}\right) \cong\left\{G \in U^{*} \mid D \circ G=0 \forall D \in\right.$ D $\}+H^{2}(\mathfrak{D})$.

We conclude with the computation of the first-order cyclic cohomology of the truncated polynomial algebra $\mathscr{O}_{n}$. We use multi-index notation: $\Gamma_{n}:=\{\alpha=$ $\left.\left(\alpha_{1}, \ldots, \alpha_{n}\right) \in \mathbb{Z}^{n} \mid 0 \leq \alpha_{i}<p\right\}, x^{\alpha}=x_{1}^{\alpha_{1}} \cdots x_{n}^{\alpha_{n}}, \varepsilon_{i}$ denotes

$$
\left(0, \ldots, \frac{1}{(i)}, \ldots, 0\right) \text {. }
$$

Proposition 3.3. $\operatorname{dim} H C^{1}\left(\mathscr{O}_{n}\right)=(n-1) p^{n}+1$. Each cocycle is of the form

$$
x^{\alpha} \wedge x^{\beta} \mapsto \sum_{i=1}^{n} \alpha_{i} \lambda_{i}\left(\alpha+\beta-\varepsilon_{i}\right)
$$

where $\lambda_{i}(\alpha) \in \mathbb{F}$ are parameters satisfying the relations

$$
\sum_{i=1}^{n} \alpha_{i} \lambda_{i}\left(\alpha-\varepsilon_{i}\right)=0
$$

for any $\alpha \in \Gamma_{n}$. 
Proof. From the computational point of view it is more convenient to consider cyclic homology. Since $\mathscr{O}_{n}$ is commutative, $H C_{1}\left(\mathscr{O}_{n}\right) \cong \Omega_{\mathscr{O}_{n}}^{1} / d \mathscr{O}_{n}$, where $\Omega_{\mathscr{O}_{n}}^{1}$ is the module of Kähler differentials (cf. [5,9]). It is easy to see that each element $u d v, u, v \in \mathscr{O}_{n}$, may be represented as a sum of elements of kind $x^{\alpha} d x^{\varepsilon_{i}}$. Moreover, we have

$$
0=d x^{\alpha}=\sum_{i=1}^{n} \alpha_{i} x^{\alpha-\varepsilon_{i}} d x_{i}
$$

for each $\alpha \in \Gamma_{n}$. So there are $n p^{n}$ generators of the vector space $H C_{1}\left(\mathscr{O}_{n}\right)$, and $p^{n}-1$ linear relations between them (if $\alpha=0,(23)$ gives a trivial equality). Hence $\operatorname{dim} H C_{1}\left(\mathscr{O}_{n}\right)=(n-1) p^{n}+1$.

Because of the duality isomorphism $H C^{1}\left(\mathscr{O}_{n}\right) \cong H C_{1}\left(\mathscr{O}_{n}\right)^{*}$, the first assertion of the lemma holds.

Further, it is easy to verify that the cochains, defined by (21) ad (22), are really cocycles. Since there are $n p^{n}$ parameters and $p^{n}-1$ relations between them, the dimension equality implies that all cocycles are taken into account.

Given a concrete subalgebra $\mathscr{D}$, it is easy to compute $H C^{1}(U)^{\mathscr{D}}$. In the general case we are able to give a "lower bound" only:

Proposition 3.4. $\operatorname{dim} H C^{1}\left(\mathscr{O}_{n}\right)^{\operatorname{Der}\left(\mathscr{O}_{n}\right)}=n p^{n-1}$.

Each $\operatorname{Der}\left(\mathscr{O}_{n}\right)$-invariant cocycle is determined by formula (21), where

$$
\lambda_{i}(\alpha)=0 \quad \text { if } \alpha_{i} \neq p-1 \text {. }
$$

Proof. $\operatorname{Der}\left(\mathscr{O}_{n}\right)$ has as a basis $\left\{x^{\gamma} \partial / \partial x_{j} \mid \gamma \in \Gamma_{n}, \quad 1 \leq j \leq n\right\}$. Writing the $x^{\gamma} \partial / \partial x_{j}$-invariance condition for the cocycle determined by formula (21), we get

$$
\sum_{i=1}^{n}\left(\alpha_{i} \beta_{j}-\alpha_{j} \beta_{i}\right) \lambda_{i}\left(\alpha+\beta+\gamma-\varepsilon_{i}-\varepsilon_{j}\right)=0 .
$$

Setting here $\beta=\varepsilon_{j}$ and $\gamma=0$, we obtain $\alpha_{j} \lambda_{j}\left(\alpha-\varepsilon_{j}\right)=0$, which is equivalent to (24). Obviously, the last condition implies (22) and (25).

In particular, $H C^{1}\left(\mathscr{O}_{1}\right)^{\mathscr{D}}=H C^{1}\left(\mathscr{O}_{1}\right)$ for any $\mathscr{D} \subseteq \operatorname{Der}\left(\mathscr{O}_{1}\right)$.

\section{ACKNOWLEDGMENT}

I am deeply grateful to A. S. Dzhumadil'daev for friendly support and stimulating discussions.

Note added in proof. Recently A. Haddi (Comm. Algebra 20 (1992), 11451166) has obtained the general formula expressing the homology group $H_{2}(L \otimes A)$ in terms of $H_{2}(L)$ and $H C_{1}(A)$ for arbitrary Lie algebra $L$ in the case of characteristic zero.

\section{REFERENCES}

1. R. E Block, On the Mills-Seligman axioms for Lie algebras of classical type, Trans. Amer. Math. Soc. 121 (1966), 378-392.

2. __ On the extensions of Lie algebras, Canad. J. Math. 20 (1968), 1439-1450.

3. __ Determination of the differentiability simple rings with a minimal ideal, Ann. of Math. 90 (1969), 433-459. 
4. N. Bourbaki, Groupes et algèbres de Lie, Chapitres VII, VIII, Hermann, Paris, 1975.

5. P. Cartier, Homologie cyclique: rapport sur les travaux récents de Connes, Karoubi, Loday, Quillen..., Astérisque 121-122 (1985), 123-148.

6. A. S. Dzhumadil'daev, Central extensions of the Zassenhaus algebra and their irreducible representations, Math. USSR-Sb. 54 (1986), 457-474.

7. D. B. Fuchs, Cohomology of infinite-dimensional Lie algebras, Consultants Bureau, New York, 1986.

8. A. Haddi, Détermination des extensions centralees des algèbres de Kac-Moody, C. R. Acad. Sci. Paris 306 (1988), 691-694.

9. C. Kassel, Kähler differentials and coverings of complex simple Lie algebras extended over a commutative algebra, J. Pure Appl. Algebra 34 (1984), 265-275.

10. M. I. Kuznetsov, The modular simple Lie algebras with a solvable maximal subalgebra, Math. USSR-Sb. 30 (1976), 68-76.

Pushkina 75, kv.40, Alma-Ata 480100, USSR

Current address: Department of Mathematics, Bar-Ilan University, Ramat-Gan 52900, Israel

E-mail address: zusman@bimacs.cs.biu.ac.il 